\title{
SUPRIMENTO ARTERIAI, DO TIMO EM CÃES SRD
}

\author{
THE ARTERIAL SUPPLY OF THE THYMUS IN DOGS
}

\begin{abstract}
Frederico ()zanan (arneiro e SII, VA'; Pedro Primo BOMBONATO'; Renato Souto SEVERINO'; Sérgio Salazar DRLMMOND'; André luds Quagliato SANTOS': Maurício BORGES ${ }^{4}$; Cláudio COSTA
\end{abstract}

\begin{abstract}
RESUMO
Estudou-se em 30) căes SRD. fetos e natimontos, a origem. o número e a ordenação dos vasos tímicos utilizando-se da dissecção de peças injetadas com solução de Neoprene látex $4.50 \mathrm{e}$ lixadas em solução de formol a $10 \%$. Os resultados permitiram concluir cue a irrigação do timo é fecita por ramos diretos e indiretosoriundos das antérias torácicas internas, tronco braquiocéfálico. artérias pericardicofrênicas, troncos costocervicais e artérias subclívias. que combinadas entre si. emitiam de cinco a catorze contribuiçöes, que por sua vez evidenciaram modalidades de vascularizaçăo própria para cada peça.
\end{abstract}

UNITERMOS: Anatomia animal; Cães; Timo: Artérias

\section{INTRODUÇÃO}

Classicamente o timo é descrito como um órgão de coloração cinza amarelado pálido que no cão localiza-se no espaço mediastínico cranial, entre os dois pulmões. Sua extremidade caudal é moldada na superfície cranial do pericárdio, enquanto que cranialmente pode estender-se para além da borda cranial do $1^{2}$ par de costelas, ventralmente à traquéia. $A$ porção torácica esquerda do timo é normalmente maior que a direita. No seu desenvolvimento máximo se estende entre a $1^{a} \mathrm{e} 6^{\mathrm{a}}$ costelas, sendo a porção cranial do timo fïna e estreita, quando existente, segundo EVANS; CHRISTENSEN's (1979).

As respostas imunes que se seguem a uma exposição à presença de material estranho são executadas pelas células linfóides, que estão disseminadas por lodo o corpo, os chamados órgãos linlóides periféricos; no entanto, o desenvolvimento normal e a manutenção desses órgãos estão na dependência do timo. Animais que sofrem timectomia neonatal têm o número de linfócitos circulantes diminuídos e acentuada depressão na capacidade de efetuar alguns tipos de resposta imune. conforme nos informa a literatura clássica.

O interesse dos pesquisadores pela morfologia desse órgão só surgiu a partir da caracterização e diagnóstico das doenças denominadas auto-imunes, já que a maioria destas está de alguma forma vinculada ao timo.
Motivados pela modernidade do tema, propusemo-nos a iniciar estudos especificamente pela vascularização arterial do timo de cães, dada a sua importância.

De sorte que, nesta oportunidade objetivamos estudar o suprimento arterial do timo, de fetos e natimortos, em cães sem raça definida, principalmente no que se referc a origem, número e ordenação dos vasos tímicos, visando obter com isto subsídios para o conhecimento do órgão bem como para o aprimoramento da anatomia comparativa.

No que diz respeito à irrigação do timo cm cães, a literatura por nós consultada ć escassa, visto que os autores quando se reportam ao assunto fazem-no de mancira genérica. Já os especialistas que trabalharam com a irrigação do timo, encontramo-los somente fazendo menção aos grandes e pequenos ruminantes.

Assim, BRADLEY; GRAHAME2 (1948) relatam a irrigação do timo a partir das artérias torácicas internas, enquanto outros autores como BOSSI' (s.d.), BRUNI; ZIMMERI.' (1951), SCHWARZE; SCHRODER" (1972), SISSON;

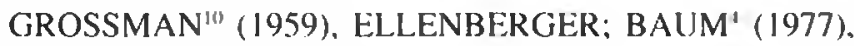
referem-se de lorma genérica à irrigação do timo, onde não especificam o animal ou não abordam o assunto relativo aos

I Professor Titular - Universidalde federal de t'herlândia-MG;

2-Professor Doutor - Fádeuldade de Medicina Veterinária c Zoroteconia dal LISP

3-Professor Assistente - Univervidade Federial de U/herländia-MCi

4-Académico - Bolsista do C.NPY
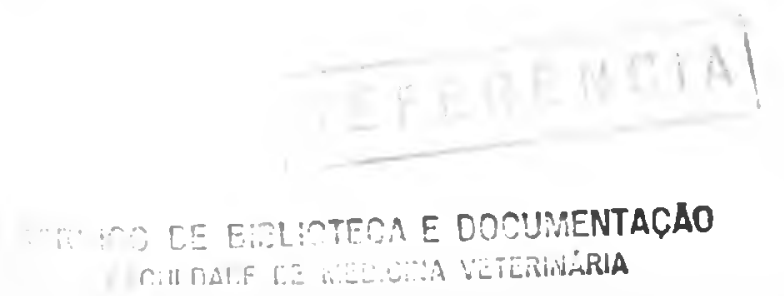


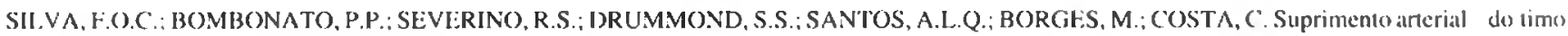
en cães SRI). Braz. J. vet. Res. anin. Sci., São Paulo, v. 31, n.2, p. 89-94, 1994.

cães, tratando principalmente da irrigação do timo $\mathrm{cm}$ bovinos e eqüinos.

EVANS; DELAHUNTA ${ }^{6}$ (1980) afirmam que o suprimento sanguíneo do timo de cães é feito através de ramos tímicos das artérias torácicas internas direita e esquerda, pericardicofrênica, primeira intercostal e troncos costocervical c braquiocefálico.

Para GETTY ${ }^{7}$ (1981) o timo de cães é irrigado por ramos das arlérias torácicas interna c timofrênica, esta oriunda do tronco braquiocefálico. Na ausência deste vaso o timo recebe suprimento sanguíneo da artéria torácica interna esquerda.

Autores especializados em anatomia de cão como EVANS; CHRISTENSEN' (1979) citam ramos tímicos oriundos das artérias torácicas internas (direita e esquerda) como sendo as principais artérias responsáveis pela irrigação do timo. Segundo eles, ocasionalmente o timo recebe ramos do tronco braçuiocefálico do lado direito e subclávia do lado esquerdo. ()utros autores que dedicaram seus estudos especificamente ao timo, c cm especial ao dos carnívoros, entre eles SANTOS et al. ${ }^{8}$ (1988), que realizaram suas pesquisas em fetos de gatos, afirmam que nestes animais o órgão recebe ramos oriundos das artérias torácica interna direita, torácica interna esquerda, subclávia esquerda e tronco braquiocefálico.

\section{MATERIAL E MÉTODO}

Para a realização deste trabalho, utilizamos 30 fetos e natimortos de cães sem raça definida, sendo 17 machos e 13 fêmeas, cujos comprimentos variavam de $12,3 \mathrm{~cm}$ a $21,2 \mathrm{~cm}$ (Tab. 1) medidas essas tomadas a partir da crista nucal até a articulação entre a $I^{\underline{a}}$ e $2^{\underline{a}}$ vértebras coccígeas.

() material era originado de abortos, mortes naturais de fêmeas prenhas e natimortos, coletados no Hospital Veterinário da Lniversidade Federal de Uberlândia, nas clínicas de pequenos animais da referida cidade $\mathrm{c}$ do Centro de Zoonoses local. As peças assim coletadas eram preparadas imediatamente após a obtenção ou conservadas em congeladores. Nestes casos, o descongelamento processava-se em água corrente por um período mínimo de 24 horas.

A pós a colcta, procedíamos a abertura da cavidade abdominal de cada animal. para a injeção de solução marcadora do vaso sanguíneo via artéria aorta abdominal. A cavidade abdominal era atingida mediante incisões na linha mediana ventral, caudalmente ao processo xifóide do osso externo, c outra, a partir dessa, de sentido ventrodorsal, margeando o arco costal e borda caudal da última costela, pelo lado esquerdo, até o processo transverso da primeira vértebra lombar, onde era feita incisão paralela à primeira que abria em janela a cavidade. Depois de identificarmos a artéria aorta, esta cra
TABELA 1

Relação de fetos e natimortos de cães Sem Raça Definida. utiliza dos no estudo do suprimento arterial do timo, segundo o sexo comprimento $(\mathrm{cm})$. Uberlândia-MG. 1992.

\begin{tabular}{|c|c|c|}
\hline $\begin{array}{c}\text { características } \\
\text { obs. }\end{array}$ & sexo & comprimento \\
\hline 1 & f & 18.0 \\
\hline 2 & $m$ & 19.7 \\
\hline 3 & $\mathrm{~m}$ & 18.4 \\
\hline 4 & $m$ & 19.1 \\
\hline 5 & $\mathrm{~m}$ & 17.1 \\
\hline 6 & $\mathrm{~m}$ & 17,5 \\
\hline 7 & $\mathrm{~m}$ & 20.1 \\
\hline 8 & $\mathrm{~m}$ & 19.6 \\
\hline 9 & f & 19,4 \\
\hline 10 & $f$ & 14.5 \\
\hline 11 & $\mathrm{~m}$ & 16.7 \\
\hline 12 & f & 17.1 \\
\hline 13 & $\mathrm{~m}$ & 19.3 \\
\hline 14 & $m$ & 17.3 \\
\hline 15 & f & 16.1 \\
\hline 16 & i & 12.3 \\
\hline 17 & f & 19.6 \\
\hline 18 & f & 18,7 \\
\hline 19 & f & 19.6 \\
\hline 20 & f & 17.1 \\
\hline 21 & f & 20,9 \\
\hline 22 & $\mathrm{~m}$ & 21,3 \\
\hline 23 & $\mathrm{~m}$ & 19.8 \\
\hline 24 & $m$ & 20,4 \\
\hline 25 & $m$ & 19.3 \\
\hline 26 & $f$ & 20.0 \\
\hline 27 & $\mathrm{~m}$ & 20.6 \\
\hline 28 & $\mathrm{~m}$ & 20.2 \\
\hline 29 & f & 20.9 \\
\hline 30 & $\mathrm{~m}$ & 20.2 \\
\hline
\end{tabular}

devidamente dissecada e mediante incisão em sua parede cra canulada, sendo a seguir injetada com solução de Neoprene látex "450" corado com pigmento específico (Du Pont do Brasil S.A. - Indústria Química), em sentido cranial. Posteriormente, as peças eram fixadas, por imersão. em soluçāo aquosa de formol a $10 \%$, por no mínimo 48 horas, para ulterior dissecação.

Para análise e descrição dos resultados, desenhos esquemáticos foram confeccionados e algumas peças fotografadas para ilustração. Os resultados assim obtidos foram submetidos à análise estatística com auxílio do teste $X^{2}$ e testada sua correlação, pelo teste de correlação de Pearson com nível de significância de $1 \%$. 
SIL.VA, F.O.C.; BOMBONATO. P.P.: SFVERINO.R.S.: DRLMMONI), S.S.; SANTOS, A.L.Q.; BORGES. M.; COSTA. C. Suprinemto anterial do timo em cäes SRD. Brał. J. vet. Res. anim. Sci., São Paulo, v. 31, n.2. p. 89-94. 1994.

\section{RESULTADOS}

Verificamos que a vascularização do timo é realizada mais frequientemente - 28 vezes $(9,3,3,3 \%: 4,5)$ a cuslas de ramos dirctos e indiretos das artérias torácicas interna direita e esquerdat. àts quais se associam eolaterais diretos e indiretos do tronco braquiocefálico - 26 vezes $(86.66 \% \pm 6.2)$; da artéria pericardicofrênica direita - 15 vezes $(50 \% \pm 9.1)$ : da artéria pericardicof rênica esquerda - 9 veces $(30 \% \pm 8,4)$; do tronco costocervical esquerdo -3 ve\%es $(10 \% \pm 5,5)$; da artéria subclávia esquerda -2 ve\%es $(6,66 \% \pm 4.5)$; da artéria subclávia direita - 1 ve $(3.3 .3 \% \pm 3.3)$ edotronco costocervical direito -1 vę $(3.33 \% \pm 3.3)$. Já, em 2 oportunidades $(6,66 \%$ \pm 4.5 ) o timo mostra-se irrigado exclusivamente por ramos diretos e indiretos das artérias torácicas internas esquerda e direita (Tab. 2).

\section{TABELA 2}

()igem das antérias destinadas ao timo de eāes Sem Raça Definidá. independentemente do seu número e tipo. Uberlândia-MG, 1992.

origem

obs.

vasos

\begin{tabular}{|c|c|c|c|c|c|c|c|c|c|}
\hline 1 & TII) & TIE & $\mathrm{TBQ}$ & $P C H D$ & PCI:L: & $\mathrm{TCCD}$ & - & . & - \\
\hline 2 & TID & TIE & $\mathrm{TBQ}$ & - & - & - & - & - & - \\
\hline 3 & TID & TIE & - & $\mathrm{PC} \cdot \mathrm{D})$ & - & - & ICCE & SE & - \\
\hline$\rightarrow$ & TID & TIF & TBQ & - & - & - & - & - & - \\
\hline 5 & TID & TIE: & TBQ & - & - & $T C(1)$ & - & - & - \\
\hline 6 & TID & ?IF & TBQ & - & PCF: & $r(C I)$ & - & - & - \\
\hline 7 & TID & TIE & TBQ & PCFD & $=$ & - & - & - & - \\
\hline 8 & TID & TIE & $T B Q$ & - & - & $\mathrm{TCCD}$ & TCCE & - & - \\
\hline 9 & TID & TIE & TBQ & PCFD & - & - & TCCE & - & - \\
\hline 10 & TID & TIF & TBQ & - & - & - & - & - & \\
\hline 11 & TII & TIE & IBQ & PCIVD & PCFL: & & - & - & - \\
\hline 12 & TID & TIE & TBQ & PCFD & PCFE: & - & - & - & $\cdot$ \\
\hline 1.3 & TID & TIF & TBQ & $\mid x(1 \% 1)$ & PCFE: & - & - & & - \\
\hline 12 & TII) & TIE: & - & $P C I D$ & $\mathrm{PC}+\mathrm{E}$ & - & - & . & . \\
\hline 15 & TII) & TIE: & $\mathrm{T} B \mathrm{Q}$ & $P(I: 1)$ & PCFE & - & - & - & - \\
\hline 16 & $T(I)$ & TIF: & TKQ & - & PCFE: & $\mathrm{T}(\mathrm{Cl})$ & & . & $=$ \\
\hline 17 & TII) & TIF: & THO & $P(1: 1)$ & - & - & & - & - \\
\hline 18 & TII) & TIE: & TBQ & - & - & - & & - & SD \\
\hline 19 & TID & TIF: & TBQ & - & . & - & - & - & - \\
\hline 20 & TII) & TIF: & TВQ & - & . & $T(C)$ & - & & - \\
\hline 21 & TII) & Tit: & TBQ & $P(I+D)$ & - & $\Gamma(C D)$ & $=$ & & - \\
\hline 22 & TID & TIl: & TBQ & PCFD & - & - & . & . & - \\
\hline 23 & TID & TIE & TBQ & - & - & $\mathrm{T}(\mathrm{CI})$ & & - & - \\
\hline 24 & TID & TIE: & $\Gamma \mathrm{BQ}$ & $P(I / D)$ & - & - & - & - & - \\
\hline 25 & TID & TIF & - & - & - & - & - & & , \\
\hline 26 & TII & TIF: & - & - & - & & - & - & - \\
\hline 27 & TID & TIE: & TIBQ & - & - & - & - & - & - \\
\hline 28 & TID & TIF. & THQ & $\mathrm{I}^{x}(\mathrm{FI})$ & - & TC(1) & - & - & - \\
\hline 29 & TII) & TIF: & 'THQ & $P(F I)$ & PCHE: & $\cdot$ & - & - & - \\
\hline 30 & TID & TII: & THQ & - & - & $\mathrm{T}(\mathrm{C}(\mathrm{I})$ & & SE & - \\
\hline
\end{tabular}

Quanto ao número de colaterais originados das referidas artérias, visualizamos a artéria torácica interna direita oferecendo um ramo - 11 vezes $(35,66 \% \pm 8,8)$. dois - 17 vezes $(56.66 \% \pm 9,1)$. ou quatro -2 veres $(6,66 \% \pm 4,5)$ todos diretos. e ainda um ramo indireto - 11 vezes $(36,66 \% \pm 8.8)$. Quando da participação da artéria torácica interna esquerda esta emite um ramo - 2 vezes $(6,66 \% \pm 4.5)$, dois - 11 vezes $(36.66 \% \pm 8.8)$, três -1.3 vezes $(43.33 \% \pm 9.1)$, quatro -3 vezes $(10 \% \pm 5,5)$ ou cinco - 1 vez $(3,3,3 \% \pm 3,3)$ ramos diretos e ainda um ramo - I l vezes $(36,66 \% \pm 8,8)$ ou dois - 1 ve\%. $(3,33 \% \pm 3.3)$ vasos indirctos.

No que tange aos vasos que se juntam às artérias torácicas interna, identificamos originando do tronco bracuiocefálico um -17 vezes $(56.66 \% \pm 9.1)$. dois -7 vezes $(23,33 \% \pm 7,7)$ ou três - 2 vezes $(6,66 \% \pm 4,5)$ ramos direlos c um ramo indireto - I vez $(3.33 \% \pm 3,3)$; da artéria pericardicofrênica direita - um ramo - 11 vezes $(36,66 \% \pm 8,8)$ ou dois - 4 vezes $(13,33 \% \pm 6,2)$ todos indiretos; da artéria pericardicofrênica esquerda - um ramo - 7 vezes $(23,3,3 \% \pm 7,7)$ ou dois - 2 vezes $(6,66 \% \pm 4,5)$ todos indiretos; do tronco costocervical esquerdo um ramo - 3 vezes $(10 \% \pm 5,5)$ direto e um ramo indireto - 1 ver $(3.33 \% \pm 3,3)$; do tronco costocervical dircito um ramo - 8 vezes $(26,66 \% \pm 8.1)$ direto e um ramo - 2 vezes $(6,66 \%$ $\pm 4,5$ ) indireto da artéria subclávia direita dois ramos - $1 \mathrm{vez}$ $(3,33 \% \pm 3,3)$; da artéria subclávia esquerda um ramo - 2 veres $(6.66 \% \pm 4.5)$ indireto (Tab. 3$)$.

Independentemente da origem e das atssociaçôes que os vasos responsáveis pela nutrição do timo possuem, esses permanecem indivisos ou ainda subdividem-se em número variável de ramos, mais precisamente: dois - 7 vezes $(23,33 \% \pm 7.7)$; três - 3 vezes $(10 \% \pm 5,5)$ e quatro - $1 \mathrm{vez}(3,3,3 \% \pm 3,3)$, para a seguir penetrarem no órgāo.

Em 11 peças - 6 machos e 5 fêmeas - $(36.66 \% \pm 8.8)$ observamos a presença de uma pequena massa tímica, dorsalmente à porção direita do órgão, sendo esta porçâo irrigada por ramos do tronco costocervical dircito e artéria pericardicofrênica direila. Assim. destas observaçóes em 8 preparaçōes $(26,66 \% \pm 8,1)$ a irrigação da relerida porção é feita exclusivamente por ramos do tronco costocervical direito. sendo que 7 vezes $(23,33 \% \pm 7,7)$ através de um ramo direto e 1 vez $(3,33 \% \pm 3,3)$ por um ramo indireto. Em outras peças - 2 veres $(6,66 \% \pm 4,5)$ o suprimento dessa porção se fez a partir da artéria pericardicofrênica dircita. Na restante observação - 1 vez $(3,33 \% \pm 3,3)$ a vascularização dessa parte do órgão é feita pela associação de um ramo do tronco costocervical direito e da artéria pericardicofrênica direita. A referida porção não foi identificada nas restantes peças - 19 vezes $(63.33 \% \pm 8,8)$. Relativamente ao número de colaterais oriundos da artéria torácica interna dircita visualizamos dois ramos - I vez $(3,3,3 \% \pm 3,3)$ e qualto - I ve\% $(3,3,3 \% \pm 3,3)$ 
SILVA.F.O.C.: BOMBONATO, P.P.: SEVERINO. R.S.: DRUMMOND, S.S.; SANTOS, A.I.Q.; BORGES, M.; COSTA.C. Suprimento arterial do timo cm cães SRD. Braz.. J. vet. Res. anim. Sci., São Paulo. v. 31. n.2, p. 89-94, 1994.

TABELA 3

Número total de artérias destinadas ao timo de cães Sem Raça Detinida, segundo sua origem. Uberlândia-MG. 1992.

vasos

obs. TID TIE TBQ PCFD PCFE TCCD TCCE SD SE total $d i d i d i \quad i \quad d i d i \quad i \quad i$

\begin{tabular}{|c|c|c|c|c|c|c|c|c|c|}
\hline 1 & $2-$ & $3-$ & $3-$ & $I$ & 1 & $1-$ & - & - & -11 \\
\hline 2 & 11 & $2-$ & $1-$ & - & - & $-\cdot$ & $-\cdot$ & - & -5 \\
\hline 3 & $4=$ & 42 & $\therefore$ & 1 & - & - & 11 & - & 114 \\
\hline 4 & 2. & $2-$ & $1-$ & - & - & - - & - & - & -5 \\
\hline 5 & $1=$ & $2-$ & $1-$ & - & - & I - & - & - & -5 \\
\hline 6 & 1. & $3-$ & $2-$ & - & 1 & I - & $\cdots$ & - & $=8$ \\
\hline 7 & $1-$ & 11 & $1-$ & 1 & - & $\cdots$ & - & - & -5 \\
\hline 8 & $2-$ & 51 & $2-$ & - & - & $1-$ & $1-$ & - & -12 \\
\hline 9 & 2 & 31 & 2 & 1 & - & - & $\cdots$ & - & -9 \\
\hline 10 & 2 & 21 & $2-$ & - & - & - & $1-$ & - & - 8 \\
\hline 11 & $1-$ & 1 & $1-$ & 1 & 2 & $\therefore$ & $\therefore$ & - & -6 \\
\hline 12 & 1. & $3-$ & $1-$ & 1 & 1 & - & - & - & -7 \\
\hline 13 & 11 & $3-$ & $1-$ & 2 & 1 & - & - & - & -9 \\
\hline 14 & 21 & $3-$ & $\therefore$ & 2 & 2 & - & $\cdot \cdot$ & $\cdot$ & $\cdot 10$ \\
\hline 15 & $1-$ & 3 & $1-$ & 2 & 1 & - & - & - & -8 \\
\hline 16 & $2-$ & 2 & $1-$ & - & 1 & $1-$ & $\cdots$ & & - 7 \\
\hline 17 & $2-$ & $2-$ & I - & 1 & - & - & - & - & -6 \\
\hline 18 & 21 & 21 & $1=$ & - & - & - & $\because$ & 2 & - 9 \\
\hline 19 & $2-$ & $2-$ & $1-$ & - & - & - & - & - & -5 \\
\hline 20 & $2-$ & $3-$ & $2-$ & - & - & -1 & $-\quad-$ & - & - 8 \\
\hline 21 & 11 & 21 & $1-$ & 2 & - & $1-$ & - - & - & -9 \\
\hline 22 & 11 & 41 & 31 & 1 & - & -- & - & - & -12 \\
\hline 23 & 1. & $3-$ & $1-$ & - & - & -1 & - & - & -6 \\
\hline 24 & $2-$ & $2-$ & $2-$ & 1 & - & - & - & - & -7 \\
\hline 25 & $2-$ & $3-$ & - & - & - & - & - - & - & -5 \\
\hline 26 & 41 & $3-$ & - & - & - & - & - & - & -8 \\
\hline 27 & 21 & 31 & 1 & - & - & - & - & - & -8 \\
\hline 28 & 21 & 21 & $1-$ & I & - & I - & - - & - & -9 \\
\hline 29 & 21 & 41 & $1-$ & 1 & 1 & $\therefore$ & - - & - & -11 \\
\hline 30 & 21 & 31 & $2-$ & - & - & $1-$ & - - & - & $\begin{array}{ll}1 & 11\end{array}$ \\
\hline
\end{tabular}

\section{ABREVIATURAS}

$\mathrm{cm}$ - Centímetro

d - Ramo direto

f - Fêmea

i - Ramo indireto

m - Macho

Obs. - Observação(ões)

PCFD - Anéria pericardicofrênica direita

PCFE - Artéria pericardicofrênica esquerda

SD - Artéria subclávia direita

SE - Artéria subclávia esquerda

SRD - Sem raça definida

TBQ - Tronco braquiocefálico

TCCD - Tronco costocervical direito

TCCE - Tronco costocervical esquerdo

TID - Artéria torácica interna direita

TIE - Artéria torácica interna esquerda diretos e um ramo indireto - 1 vez $(3,33 \% \pm 3,3)$. Quanto à artéria torácica interna esquerda, nas duas peças $(6,66 \% \pm$ 4,5 ) observamos três ramos diretos. Esses vasos permanecem indivisos ou se dividem em dois ramos - 2 vezes $(6,66 \% \pm 4,5)$ ao penetrarem no órgão.

Independentemente da origem e do tipo de ramo, observamos de 5 a 14 vasos responsáveis pela nutrição arterial do timo, mais precisamente cinco - 6 vezes $(20 \% \pm 7.3)$, oito e nove5 vezes $(16,66 \% \pm 6,8)$ cada, seis, sete e onze - 3 vezes $(10 \%$ $\pm 5,5)$ cada, doze e catorze -2 vezes $(6,66 \% \pm 4,5)$ cada e dez $-1 \operatorname{vez}(3,33 \% \pm 3,3)$.

Analisando estatisticamente a participação dos diferentes vasos que contribuem para a irrigação do timo, evidenciamos, com auxílio do teste $X^{2}$, ao nível de significância de 0,05 , que não existem diferenças significantes, quando confrontados os sexos.

Ainda, valendo-nos do teste de Correlação de Pearson. com nível de significância de $1 \%$, encontramos $\mathrm{r}=0,151374$ para a correlação entre o número total de ramos em sua origem e o comprimento corpóreo, dados estes indicativos de correlação positiva, evidenciando entrctanto baixa tendência conforme traçado da reta correspondente (Fig. 1).

O tamanho médio dos animais examinados foi de 18.6933 $\mathrm{cm}$, com mediana de $19,35 \mathrm{~cm}$, variância de $4,07306 \mathrm{e}$ erro padrāo de 0,368568 . Já em relação ao número total de vasos endereçados ao timo notamos média de 8,1 . mediana de 8 , variância de 5,95517 e erro padrão de 0.44554 .

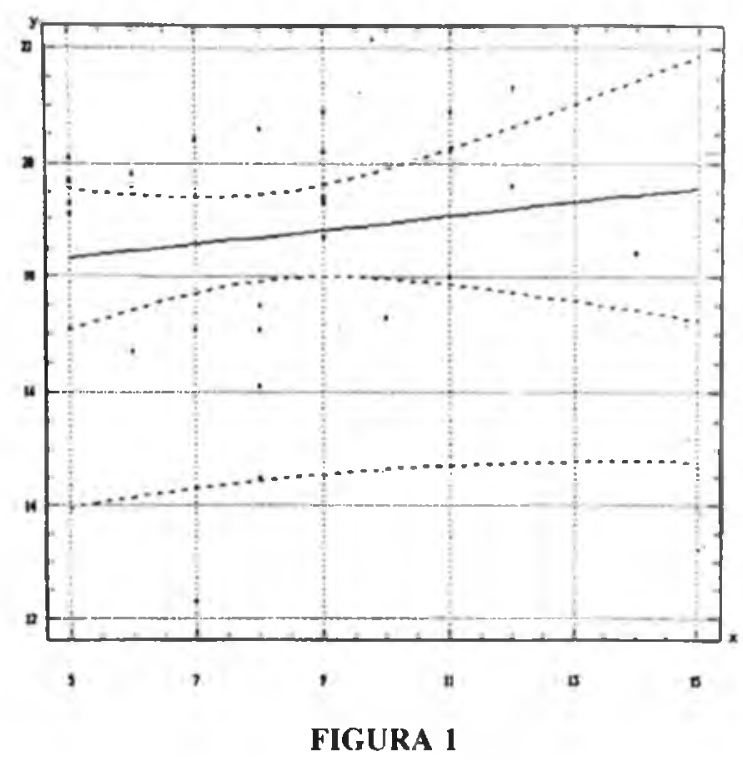

Gráfico representativo da correlação (regressão linear) entre o número total de vasos tímicos $(x)$ em relação ao comprimento corporeo (y) em cm. Uberlândia - MG, 1992. 
SILVA, F.O.C.; BOMBONATO, P.P.; SEVERINO, R.S.; DRUMMOND, S.S.; SANTOS, A.L.Q.; BORGES, M.; COSTA, C. Suprimento arterial do timo em cães SRD. Braz. J. vet. Res. anim. Sci., São Paulo, v. 31, n.2, p. 89-94, 1994.

\section{DISCUSSÃo}

O interesse pela pesquisa macroscópica do timo tem aumentado em virtude da diversidade do tema e pela escassa literatura relativa a esse órgão, de maior importe do sistema imunitário dos animais. Dados relativos à vascularização arterial do timo de cães são incompletos.

Assim, os principais tratadistas de anatomia veterinária, como BOSSI ${ }^{1}$ (s.d.), BRUNI; ZIMMERL ${ }^{3}$ (1951), SCHWARZE; SCHRODER $^{9}$ (1972), SISSON; GROSSMAN ${ }^{10}$ (1959), ELLENBERGER; BAUM ${ }^{4}$ (1977), se ocuparam, praticamente, do estudo de grandes animais (bovinos e eqüinos), onde os subsídios sobre a anatomia do timo são maiores. Entretanto, devemos ressalvar que o timo dos carnívoros possui particularidades, principalmente em relação à sua localização exclusivamente torácica, comprovada por essa pesquisa, bem como a presença de pequena massa, e que se destaca da massa principal, que conferem individualidades para esta espécie, fazendo com que haja dificuldades na generalização dos conhecimentos para as outras espécies e vice-versa.

Dentre os carnívoros, os caninos apresentam o timo proporcionalmente maior que os felinos, consequentemente apresentam também uma vascularização mais diversificada como ressalta a literatura. No entanto, a clássica descrição merece maior atenção, pois em algumas de nossas preparações $(36,66 \%)$ foi identificada uma massa tímica arredondada, paralela à porção direita, caudalmente à origem da artéria vertebral e tronco costocervical, fato este não citado pelos autores consultados, talvez por se tratar de uma estrutura de difícil observação, ou pela freqüência da sua presença.

O confronto de nossos resultados com aqueles contidos na literatura fica restrito a uma superficial análise da origem dos vasos endereçados ao timo, não só porque a literatura nos traz dados incompletos relativos aos carnívoros, como também em virtude do tamanho significantemente maior do timo dos cães do que dos gatos, aonde dispomos de alguns dados. Assim, pudemos identificar número variado e maior de vasos em comparação com o timo de gatos (SANTOS et al. ${ }^{8}$, 1988).

Onosso relato aproximou-se daqueles observados por EVANS; CHRISTENSEN $^{5}$ (1979), que afirmam que o timo é irrigado por ramos das artérias torácicas internas, do tronco braquiocefálico do lado direito e subclávia do lado esquerdo, e são descritos por EVANS; DELAHUNTA ${ }^{6}$ (1980) que responsabilizam as artérias torácicas internas, tronco braquiocefálico, pericardicofrênica, troncos costocervical e primeira intercostal, esta última não observada em nossas preparações. SANTOS et al. ${ }^{8}$ (1988) relatam que encontraram, no timo de gatos, ramos das artérias torácicas internas, tronco braquiocefálico e subclávia esquerda, resultado seme- lhante em parte ao verificado no nosso material.

Genericamente encontramos ramos tímicos oriundos principalmente da artéria torácica interna direita, torácica interna esquerda, tronco braquiocefálico, pericardicofrênica direita, pericardicofrênica esquerda, e ocasionalmente do tronco costocervical esquerdo, costocervical direito, subclávia esquerda e subclávia direita. Estes resultados coincidem parcialmente com as informações exaradas pelos tratadistas que cuidam da irrigação do timo de gatos $\operatorname{GETTY}^{7}$ (1981), bem como com as daqueles que se referem genericamente ao timo de cães como BRADLEY; GRAHAME ${ }^{2}$ (1948) e GETTY ${ }^{7}$ (1981); entretanto, nenhum dos tratadistas faz menção ao tronco braquiocefálico (exceto o primeiro), pericardicofrênicas, tronco costocervical e artérias subclávias. O mesmo acontece com as considerações de EVANS; CHRISTENSEN $^{5}$ (1979), não obstante relatarem contribuição do tronco braquiocefálico e subclávia esquerda, e com EVANS; DELAHUNTA ${ }^{6}$ (1980) que não fazem menção à participação das artérias subclávias na irrigação do timo.

Um dos confrontos que podem ser realizado dos nossos resultados com os da literatura é o que se refere ao destino dos ramos tímicos oriundos do tronco braquiocefálico, onde encontramos ramos dirigindo-se frequentemente para a porção esquerda do órgão $(83,33 \%)$, raramente para a direita $(16,66 \%)$, sendo que EVANS; CHRISTENSEN ${ }^{5}$ (1979) afirmam que o timo recebe colateral do tronco braquiocefálico do lado direito, o que difere dos dados por nós observados. Ainda com relação ao tronco braquiocefálico, este em apenas uma peça $(3,33 \%)$ cedeu ramo indireto para o timo (artéria timofrênica) independentemente da presença das artérias torácicas interna direita e esquerda, que segundo relato de GETTY? (1981) está ausente quando a irrigação é feita pela artéria torácica interna esquerda, tendo o autor, talvez, feito a referência em função da frequêencia do evento.

Após a análise dos resultados, observamos que o número de vasos que irrigam o timo independe do sexo ou tamanhos dos animais à semelhança de SANTOS et al. ${ }^{8}$ (1988). Gostaríamos ainda de destacar que nos resultados por nós obtidos, o timo apresentou modalidades individuais de vascularização quando considerados o número, procedência e ordenação dos vasos a ele destinados, o que nos permite supor que as particularidades quando da involução deste órgão esteja a este fato relacionado.

\section{CONCLUSÕES}

Ao analisarmos os resultados obtidos, cremos poder chegar às seguintes conclusões:

1. o timo de cães sem raça definida é irrigado por ramos diretos c indiretos das artérias torácica interna direita, torácica 
SII,VA, F.O.C.; BOMBONATO. P.P.; SI:VI:RINO, R.S.; I)RUMMOND, S.S.: SANTOS. A.L.Q.; BOR(jE:S. M.; COSTA. C. Suprimento arterial do timo en cães SRD. Braz. J. vet. Res. anim. Ści.. São Paulo, v. 31. n. 2. p. 89-94. 1994.

interna esquerda, tronco braquiocefálico, pericardicof rênica direita, pericardicof rênica esquerda. troncocostocervical direito, tronco costocervical esquerdo, subclávia direita e subclávia esquerda:

2. independentemente da origem das artérias, o timo dos cines recebe de cinco - (20\%); oito, nove - (16,66\%); seis, sete, onze- (10\%): do\%, cator $(6,66 \%)$; de $(3.33 \%)$ vasos
3. não foram notadas diferenças estatisticamente significante relativamente aos sexos. no que tange à participação do diferentes vasos que contribucm com a vascularização at terial do timo;

4. considerando conjuntamente o número, a procedéncia e ordenação das artérias destinadas ao timo, identificamo modalidades de vascularização próprias para cada peça

\section{SUMMARY}

There were observed the origin. the number and the ordering of the thymic vessels in 30 dogs fetuses. by dissection after injection with Neoprene latex 4.50 solution and fixation with formalin solution al $10 \%$. "The results permited to conclude that: the thymic arteries come, directly and indirectly, from the internal thoracic arteries. brachiocephalic trunk. pericardicophrenics aneries. costocervicals trunks and subclavian aneries. wich combined by theirself given from five to fourteen contributions that showed a own vascularization model for each organ.

UNITERMS: Animal anatomy: Dogs: Thymus gland: Arteries

\section{REFERÊ.NCIAS BIBLIOGRÁFICAS}

()1-BOSSI, V. Angiologia. In: BOSSI, V.; CARADONA. G.B.; SPAMPANI, G.: VARALDI. L.: ZIMMERL. U. Tratado di anatomia veterinária. Milano, Francesco Vallardi, s.d. v.2, p.88.

()2-BRADLEY, O.C.; GRAHAME, T. Topographical anatomy of the dog. I6.ed. Edinburg. W. Green \& Son, 1948. p.51.

03-BRUNI, A.C.: \%IMMERL. U. Anatomia degli animali domestici. 2.ed. Milano, Francesco Vallardi, 1951. v.4, p.261.

04-ELLENBERGER. W.; BAUM. H. Handbuch der Vergleichenden Anatomie der Haustiere. 18. Auf. Berlin, Springer Verlag, 1977. p.603.

05-EVANS, H.E.; CHRISTENSEN, G.C. Miller's anatomy of the dog. 2.ed. Philadelphia. W.B. Saunders, 1979. p.839-40.

06-IVVANS, H.E.: DELAHUNTA. A. Miller's guide to the dissection of the dog. 2.ed. W.B. Saunders. 1980). v.1, p. $11.5-22$
07-GETTY. R. Anatomia dos animais domésticos. 5.ed Rio de Janeiro, Interamericana. 1981. v.2, p.1504-68

08-SANTOS, A.L.Q.: SILVA, F.O.C.: SEVERINO. R.S DRUMMONI), S.S.: BOMBONATO, P.P. Contribui ção ao estudo da vascularização arterial do timo en gatos S.R.D. In: CONGRESSO BRASILEIRO IDF ANATOMIA. 15. Brasília, 1988. Resumos. Brasília Universidade de Brasília, 1988 . p.109.

(0)-SCHWARZE:, E.: SCHRODER. I. Compêndio de ana tomia veterinária: aparato circulatório y piel Zaragoza, Acribia, 1972. v.3, p.182.

10-SISSON, S.; GROSS.MAN, J.D.Anatomia de los animales domesticos. 4.ed. Barcelona. Salvat. 1959. p.526-43

Recebido para publicação em 13/11/92 A provado para publicação em 24/08/9? 